\title{
Distribuição comparativa dos glicosaminoglicanos em artérias e veias de diferentes mamíferos
}

\author{
Mônica V. MARQUEZINI*, Luís Alberto O. DALLAN**, Olga M. S. TOLEDO***
}

RBCCV 44205-474

\begin{abstract}
Marquezini M V, Dallan L A O, Toledo O M S - Distribuição comparativa dos glicosaminoglicanos em artérias e veias de diferentes mamíferos. Rev Bras Cir Cardiovasc 1999; 14 (4): 325-30.

RESUMO: Foi realizada análise comparativa sobre a distribuição dos glicosaminoglicanos de artérias e veias em ratos, cachorros e humanos. Os nossos resultados demonstraram que dermatam sulfato foi 0 principal glicosaminoglicano encontrado tanto para as artérias quanto para as veias estudadas. Entretanto, a proporção de dermatam sulfato foi maior nas veias do que nas artérias nas três espécies analisadas. Este aumento pode estar associado às diferenças estruturais e funcionais encontradas na parede destes dois tipos de vasos sangüíneos (nas veias a pressão sangüínea é significativamente mais baixa). Além disso, a quantidade total dos glicosaminoglicanos foi maior nas artérias do que nas veias, sendo as maiores concentrações encontradas nas aortas independentemente da espécie animal estudada. Estes achados abrem perspectiva para o melhor conhecimento das alterações das macromoléculas que possam estar relacionadas ao processo degenerativo vascular, especialmente nas transformações estruturais que as veias safenas sofrem, quando empregadas como enxertos na revascularização do miocárdio.
\end{abstract}

DESCRITORES: Glicosaminoglicanos, análise. Artérias, química. Veias, química.

\section{INTRODUÇÃO}

O sistema vascular apresenta características peculiares relacionadas à parede dos vasos, variando entre os de maior e menor calibre. Os vasos sangüíneos são compostos por tecidos particularmente ricos em matriz extracelular cujos componentes individuais, proteoglicanos, fibras dos sistemas elástico e colágeno, mudam como resultado de adaptações às exigências funcionais locais. Além disso, as artérias diferem funcional e estruturalmente das veias. É bem estabelecido que os dois principais tipos celulares dos vasos sangüíneos, as células endoteliais e as musculares lisas, sintetizam os proteoglicanos (1).

Nos últimos anos, a distribuição dos glicosaminoglicanos de vasos sangüíneos tem sido estudada particularmente na parede arterial, principalmente da aorta, devido ao seu envolvimento na patogênese da aterosclerose (2-8), hipertensão arterial (9) e diabetes (10-13).

Muitos são os dados na literatura sobre a distribuição e provável função dos glicosaminoglicanos em outras artérias elásticas ou musculares $(6,14-16)$.

\footnotetext{
Trabalho realizado na Pró-Sangue Fundação Hemocentro de São Paulo, no Instituto do Coração do Hospital das Clínicas da Faculdade de Medicina da Universidade de São Paulo e na Escola Paulista de Medicina - Universidade Federal de São Paulo. São Paulo, SP, Brasil.

Recebido para publicação em agosto de 1999.

* Da Pró-Sangue Fundação Hemocentro de São Paulo.

** Do Instituto do Coração do Hospital das Clínicas da Faculdade de Medicina da Universidade de São Paulo.

*** Da Escola Paulista de Medicina - Universidade Federal de São Paulo.

Endereço para correspondência: Olga M S Toledo. Escola Paulista de Medicina, Departamento de Morfologia. Rua Botucatu, 740. São Paulo, SP, Brasil. CEP: 04023-062. Telefax: (011) 576-4268/576-4271. E-mail: olmatoledo.morf@epm.br
} 
Marquezini M V, Dallan L A O, Toledo O M S - Distribuição comparativa dos glicosaminoglicanos em artérias e veias de diferentes mamíferos. Rev Bras Cir Cardiovasc 1999; 14 (4): 325-30.

Por outro lado, poucos são os trabalhos que relatam a distribuição destes compostos em veias $(14,17,18)$, e nenhum resultado sobre a análise comparativa da distribuição de glicosaminoglicanos em artérias e veias pode ser encontrado.

O conhecimento da distribuição destas macromoléculas nas diferentes veias e artérias é de considerável relevância, uma vez que a revascularização cirúrgica do miocárdio utiliza enxertos do próprio paciente, de segmentos de veias ou artérias. Dados da literatura mostram que um porcentual significativo destes enxertos será comprometido por lesões ateroscleróticas (19-26). Além disso, os enxertos venosos e arteriais diferem em relação à susceptibilidade à aterosclerose e, consequentemente, ao tempo de durabilidade do enxerto (27).

Os proteoglicanos arteriais são reconhecidamente fatores importantes no processo da aterosclerose tanto pela habilidade em seqüestrarem lipídeos para dentro da parede arterial, quanto pelo envolvimento nos processos de adesão, migração e proliferação celular $(1,28)$. Sabemos, também, que os diversos tipos de proteoglicanos têm diferentes e, muitas vezes, opostas funções.

O objetivo deste trabalho foi a análise comparativa da distribuição dos glicosaminoglicanos entre várias artérias e algumas veias de ratos, cães e humanos. Estas três espécies foram escolhidas para este propósito devido as suas, já conhecidas, diferenças no padrão de distribuição dos glicosaminoglicanos aórticos e susceptibilidade a aterosclerose (4).

\section{CASUÍSTICA E MÉTODOS}

\section{Casuística}

Vasos sangüíneos humanos normais foram obtidos de necropsias ( 9 indivíduos de ambos os sexos, na quinta década de vida) e compreenderam: a aorta torácica e as artérias: carótida, subclávia e ilíaca, além das veia cava e ilíaca.

Os vasos obtidos de ratos adultos foram aorta e cava; artéria e veia ilíacas, e os obtidos de cães foram: aorta e cava, artéria e veia ilíacas, artéria femoral e veia safena, artéria e veia pulmonares, artéria carótida e veia jugular.

As amostras de aorta e da cava consistiram em segmentos torácicos e abdominais (com exceção de humanos). As artérias e veias ilíacas foram obtidas do segmento distal da ilíaca comum e do segmento proximal da ilíaca externa e interna. Somente as camadas média e íntima foram analisadas. A camada adventícia foi previamente dissecada e retirada dos vasos.
Foram utilizados os padrões de condroitim 4sulfato, condroitim 6-sulfato, dermatam sulfato e heparam sulfato da Sigma Co Químico, St, Louis, Mo.

Tripsina (E.C. 3.4.4.4) de pâncreas bovino III, condroitinase AC-II (E.C. 4.2.5.5), extraída de Arthrobacter aurenscens, condroitinase ABC (E.C. 4.2.2.4.) extraída de Proteus vulgaris, e heparinase III ( E.C. 4.2.2.8) de Flavobacterium heparinum também foram adquiridas da Sigma Co Químico, St. Louis, Mo.

Agarose ("standard Low-Mr") foi adquirida da Bio Rad Laboratories (Richmond, CA, EUA).

\section{MÉTODOS}

Isolamento dos glicosaminoglicanos: As camadas média-íntima dos vasos sangüíneos foram cortadas em pedaços muito pequenos e deixadas por $48 \mathrm{~h}$ a $5^{\circ} \mathrm{C}$ em acetona. Em seguida, o tecido livre de lipídeos neutros e substâncias liposolúveis foi obtido por centrifugação $\left(2.000 \mathrm{~g}, 30 \mathrm{~min}\right.$ a $5^{\circ} \mathrm{C}$ ) e o precipitado resultante (pó cetônico) seco à vácuo.

Os glicosaminoglicanos foram, então, extraídos por incubação de $10 \mathrm{mg}$ de pó cetônico com $1 \mathrm{mg}$ de tripsina em tampão Tris- $\mathrm{HCl}$ 0,05M, pH 8,0 como descrito por MARQUEZINI et al. (18) em 1995.

Os vasos obtidos de cães e humanos foram submetidos a extração individualmente, enquanto que os vasos obtidos de ratos, devido ao seu pequeno tamanho, foram processados em grupos com aproximadamente 9 espécimes de cada vaso sangüíneo.

Eletroforese em gel de agarose: cerca de $10 \mu \mathrm{g}$ de glicosaminoglicanos foram aplicados em lâminas de gel de agarose a $0,5 \%$ em tampão 1,3diaminopropano-acetato a 0,05M, pH 9,0. Após corrida eletroforética (120V por $1 \mathrm{~h}$ ), os glicosaminoglicanos foram precipitados no gel com $0,1 \%$ Cetavlon (brometo de N-acetyl-N, N, N-trimethylammonium) e corados com $0,1 \%$ azul de toluidina em ácido acético/etanol/água $(0,1: 5: 5, \mathrm{v} / \mathrm{v})$. A quantificação dos glicosaminoglicanos foi realizada por densitometria das lâminas de agarose coradas com azul de toluidina (29).

Ensaio enzimático: Amostras contendo cerca de $100 \mu \mathrm{g}$ de glicosaminoglicanos foram incubadas com condroitinase AC-II $(0,01 \mathrm{U})$, condroitinase ABC $(0,01 \mathrm{U})$ ou heparinase III $(0,01 \mathrm{U})$ em tampão Trisacetato $0,05 \mathrm{M}, \mathrm{pH} 7,3$, por $24 \mathrm{~h}$ a $37^{\circ} \mathrm{C}\left(30^{\circ} \mathrm{C}\right.$ para heparitinase III).

O tratamento com a condroitinase AC-II degrada os glicosaminoglicanos com migração eletroforética semelhante ao condroitim 4/6-sulfato pa- 
Marquezini M V, Dallan L A O, Toledo O M S - Distribuição comparativa dos glicosaminoglicanos em artérias e veias de diferentes mamíferos. Rev Bras Cir Cardiovasc 1999; 14 (4): 325-30.

drão. Por outro lado, o tratamento com a condroitinase $A B C$ degrada os glicosaminoglicanos com migração semelhante ao condroitim 4/6-sulfato e dermatam sulfato, enquanto que heparitinase III degrada somente os glicosaminoglicanos com migração semelhante ao padrão de heparam sulfato.

Cromatografia em papel: Após a digestão das amostras com as condroitinases ou heparinase III, estas foram aplicadas em papel "Whatman" $n^{\circ} 1$ e submetidas a cromatografia descendente em solvente ácido isobutírico/amônia (1M) $(5: 3, v / v)$ por $18 \mathrm{~h}$. Os dissacarídeos insaturados formados foram visibilizados por nitrato de prata e quantificados através de densitometria (30).

\section{RESULTADOS}

A eletroforese em gel de agarose dos extratos de glicosaminoglicanos obtidos da artéria e veia ilíacas de cão é mostrada na Figura 1. Uma banda foi obtida correspondente ao padrão de migração de dermatam sulfato. Este padrão foi observado para todos os vasos sangüíneos estudados, com exceção dos vasos humanos e aortas de cães, onde a banda eletroforética principal correspondeu ao padrão de migração de condroitim 4/6 sulfato padrão.

Da análise da quantidade relativa das unidades dissacarídicas obtidas da degradação de condroitim 4/6 sulfato pela condroitinase AC-II observamos que as unidades 6-sulfatadas ocorreram em maior concentração do que as unidades 4-sulfatadas para todas as artérias estudadas (Tabela 1).

Nenhuma tentativa foi feita para separar os condroitim 4 - e 6-sulfatos das veias. Estes compostos foram designados como condroitim sulfato e migraram como uma banda única neste sistema de eletroforese.

As porcentagens relativas de condroitim sulfato, dermatam sulfato e heparam sulfato obtidas por densitometria do gel de agarose, assim como a quantidade total destes glicosaminoglicanos são mostradas na Tabela 2.

As duas veias humanas estudadas, a cava e a ilíaca, classificadas como veias de grande calibre, apresentaram padrão idêntico em relação às quantidades relativas de glicosaminoglicanos, sendo dermatam sulfato o componente principal (93\%) seguido por heparam sulfato $(5 \%)$ e condroitim sulfato $(\leq 2 \%)$.

Nas artérias humanas (ilíacas, carótida e subclávia) dermatam sulfato também foi o principal glicosaminoglicano encontrado. A aorta, como já bem documentada (4), apresentou condroitim sulfato como o principal glicosaminoglicano obtido (93\%).

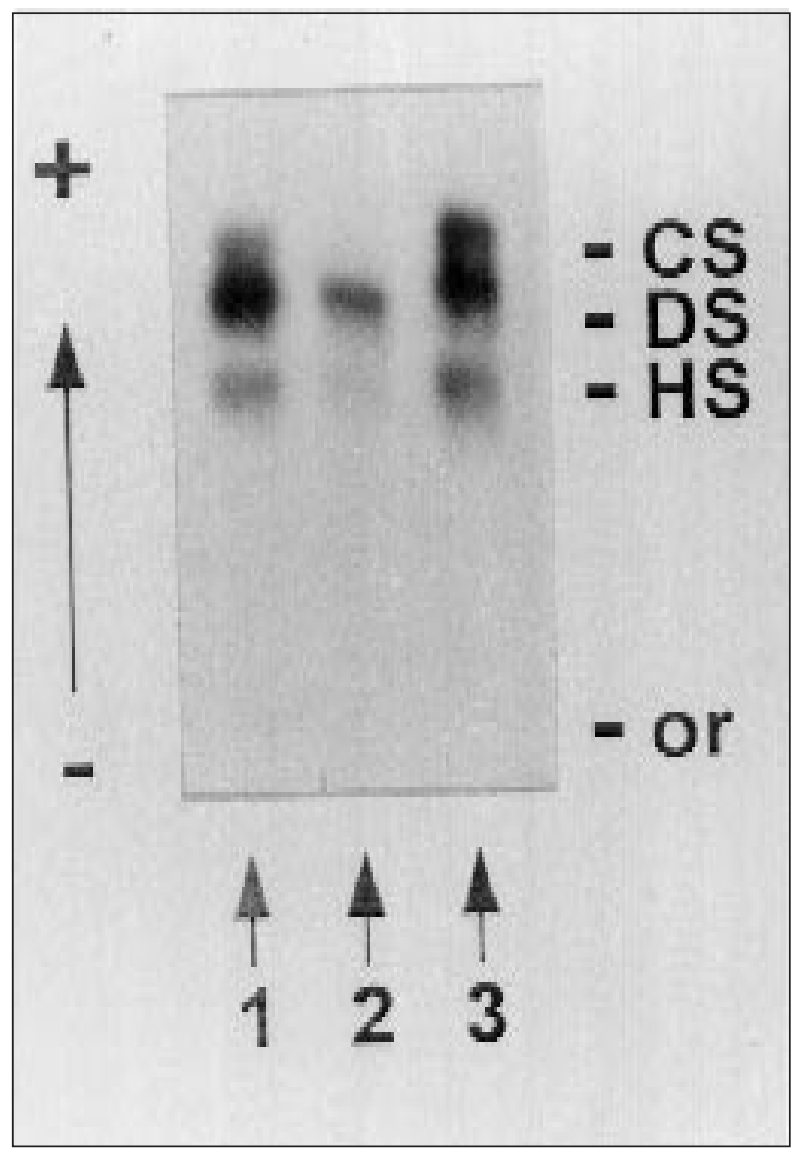

Fig. 1 - Eletroforese dos glicosaminoglicanos (GAGs) da artéria e veia ilíacas de cão. Amostras contendo cerca de $10 \mu \mathrm{g}$ of GAGs extraídos da artéria (1) e da veia ilíaca (2) foram aplicadas em lâminas de gel de agarose a 0,5\% em tampão 1,3-diaminopropano acetato, $\mathrm{pH}$ 9,0. Após a corrida eletroforética, os GAGs foram precipitados no gel com cetavlon a $0,1 \%$ e corados com azul de toluidina a $0,1 \%$ em ácido acético/etanol/água $(0,1 / 5 / 5, \mathrm{v} / \mathrm{v})$. $\mathrm{P}=$ mistura de glicosaminoglicanos padrões contendo $5 \mu \mathrm{g}$ de cada um dos compostos: condroitim 6-sulfato (CS); dermatam sulfato (DS) e heparam sulfato (HS)

Como podemos observar na Tabela 2, o padrão de distribuição dos glicosaminoglicanos foi semeIhante para artérias e veias nas três espécies estudadas. Dermatam sulfato foi o principal glicosaminoglicano encontrado. Porém, as quantidades relativas de dermatam sulfato foram maiores nas veias quando comparadas com as artérias. Por exemplo, as artérias ilíacas de cães e ratos apresentaram $58 \%$ e $53 \%$ de dermatam sulfato, respectivamente, enquanto que as veias ilíacas correspondentes mostraram $82 \%$ e $76 \%$ de dermatam sulfato, respectivamente. Também pudemos observar que, em relação as artérias de rato, a porcentagem de heparam sulfato foi aproximadamente $20 \%$ maior quando comparada com os outros vasos sangüíneos dos demais animais analisados.

Os resultados apresentados mostraram que as artérias contêm maior quantidade de glicosami- 
Marquezini M V, Dallan L A O, Toledo O M S - Distribuição comparativa dos glicosaminoglicanos em artérias e veias de diferentes mamíferos. Rev Bras Cir Cardiovasc 1999; 14 (4): 325-30.

\section{TABELA 1}

QUANTIDADE RELATIVA DOS DISSACARIDEOS INSATURADOS CONSTITUINTES DE CONDROITIM 4- (UDI4S) AND 6-SULFATOS (DDI6S) EM ALGUMAS ARTÉRIAS DE DIFERENTES MAMIIFEROS (EXPRESSAS COMO PORCENTAGEM DA QUANTIDADE TOTAL DE CONDROITIM 4/6 SULFATOS)

\begin{tabular}{lccc}
\hline MAMíFEROS & ARTÉRIAS & DDI4S (\%) & DDI6S (\%) \\
\hline Cão & Aorta & 18,0 & 40,0 \\
& Carótida & $\leq 2$ & $\leq 2$ \\
& Ilíaca & $\leq 2$ & $\leq 2$ \\
& Femoral & 6,6 & 8,4 \\
Rato & Pulmonar & $\leq 2$ & $\leq 2$ \\
Humano & Aorta & $\leq 2$ & 6,0 \\
& Aorta & 24,4 & 68,6 \\
& Ilíaca & 2,9 & 20,1 \\
& Carótida & 4,6 & 18,4 \\
& Subclavia & $\leq 2$ & 27,0 \\
\hline
\end{tabular}

As unidades dissacarídicas, obtidas após degradação com a condroitinase AC-II, foram separadas por cromatografia descendente em ácido isobutírico $\mathrm{NH}_{4} \mathrm{OH}$, seguida por coloração em nitrato de prata e quantificados por densitometria.

noglicanos quando comparadas com as veias, e entre as artérias, a aorta apresentou as maiores concentrações (Tabela 2).

\section{COMENTÁRIOS}

Pela primeira vez foi demonstrado que dermatam sulfato é o glicosaminoglicano predominante tanto nas veias quanto nas artérias, exceto para a aorta de cães e humanos.

Os nossos dados mostrando a predominância de dermatam sulfato em veias estão de acordo com os relatos prévios de IZUKA \& MURATA (17) que analisaram, em 1972, a veia cava humana (veia de grande calibre) e de MARQUEZINI et al. (18), que estudaram a veia safena humana (veia de médio calibre), em 1995.

Também pudemos demonstrar que há uma diferença significante entre a quantidade relativa de dermatam sulfato das artérias e suas veias correspondentes. As veias possuem maior concentração de dermatam sulfato. O significado funcional deste aumento não está claro. Sabemos, entretanto, que nas veias a pressão sangüínea é significantemente mais baixa e que elas exibem uma menor compressibilidade quando comparadas com as artérias.

O conhecimento da distribuição dos glicosaminoglicanos nas diferentes veias e artérias poderá trazer subsídios para o melhor entendimento do processo degenerativo vascular, tanto do sistema arterial, quanto das veias safenas quando emprega-

TABELA 2

\begin{tabular}{|c|c|c|c|c|c|c|c|}
\hline \multirow[t]{2}{*}{ MAMÍFEROS } & \multirow[t]{2}{*}{$\mathbf{N}^{*}$} & \multicolumn{2}{|c|}{ VASOS } & \multirow{2}{*}{$\begin{array}{c}{ }^{*} \text { TOTAL GAGs } \\
\mu \mathrm{g} / \mathrm{g} \text { de } \\
\text { TECIDO SECO }\end{array}$} & \multicolumn{3}{|c|}{$\%$ GAGs } \\
\hline & & ARTÉRIAS & VEIAS & & $c s$ & $D S$ & HS \\
\hline \multirow[t]{10}{*}{ Cão } & 8 & Aorta & & $14,71 \pm 1,68$ & 58 & 32 & 10 \\
\hline & & & Cava & $5,58 \pm 0,55$ & $\leq 2$ & 88 & 10 \\
\hline & & Ilíaca & & $7,55 \pm 0,94$ & 18 & 58 & 24 \\
\hline & & & Ilíaca & $3,59 \pm 0,40$ & $\leq 2$ & 82 & 16 \\
\hline & & Femoral & & $5,14 \pm 0,53$ & 13 & 67 & 20 \\
\hline & & & Safena & $3,54 \pm 0,37$ & $\leq 2$ & 93 & 5 \\
\hline & & Carótida & & $4,50 \pm 0,18$ & $\leq 2$ & 82 & 16 \\
\hline & & & Jugular & $2,86 \pm 0,26$ & $\leq 2$ & 82 & 16 \\
\hline & & Pulmonar & & $3,43 \pm 0,39$ & $\leq 2$ & 91 & 7 \\
\hline & & & Pulmonar & $1,94 \pm 0,14$ & $\leq 2$ & 93 & 5 \\
\hline \multirow[t]{4}{*}{ Rato } & 9 & Aorta & & $16,08 \pm 0,79$ & 6 & 54 & 40 \\
\hline & & & Cava & $4,48 \pm 0,40$ & 10 & 74 & 16 \\
\hline & & Ilíaca & & $5,55 \pm 0,48$ & $\leq 2$ & 53 & 42 \\
\hline & & & llíaca & $2,69 \pm 0,18$ & $\leq 2$ & 76 & 24 \\
\hline \multirow[t]{6}{*}{ Humano } & 9 & Aorta & & $19,46 \pm 1,73$ & 93 & 5 & $\leq 2$ \\
\hline & & & Cava & $4,60 \pm 0,42$ & $\leq 2$ & 93 & 5 \\
\hline & & Ilíaca & & $4,79 \pm 0,25$ & 23 & 67 & 10 \\
\hline & & & llíaca & $2,29 \pm 0,25$ & $\leq 2$ & 93 & 6 \\
\hline & & Carótida & & $5,04 \pm 0,36$ & 23 & 75 & $\leq 2$ \\
\hline & & Subclávia & & $11,56 \pm 1,24$ & 27 & 55 & 18 \\
\hline
\end{tabular}

${ }^{*} \mathrm{~N}=$ número de amostras

** Os dados são mostrados como média + desvio padrão

$\mathrm{CS}$ = condroitim sulfato; $\mathrm{DS}$ = dermatam sulfato; $\mathrm{HS}$ = heparam sulfato 
das na revascularização do miocárdio, assim como na prevenção da restenose. SCOTT et al. (27) demonstraram que $90 \%$ dos enxertos que utilizam a artéria torácica interna continuam funcionais por aproximadamente 10 anos. No entanto, 20\% dos enxertos que utilizam a veia safena tornam-se inativos já nos primeiros anos após a operação e, aproximadamente, $45 \%$ estarão seriamente comprometidos nos próximos 10 anos.

A deposição e interação de lipoproteínas na matriz extracelular das artérias é sabidamente um evento crucial no desenvolvimento da aterosclerose. A parede arterial contém uma variedade de proteoglicanos. No entanto, trabalhos sobre a interação entre lipoproteínas e proteoglicanos em suínos ${ }^{(31)}$ e humanos (32) mostraram que os proteoglicanos que intera- gem efetivamente com as lipoproteínas são aqueles que contêm dermatam sulfato na sua estrutura.

Os trabalhos de O' BRIEN et al. (33) e de PENTIKÄINEN et al. (34) mostraram que biglicam e decorim, proteodermatans sulfatos, interagem e retêm as lipoproteínas na matriz extracelular da parede arterial.

Podemos então especular que a presença de dermatam sulfato na parede dos vasos sangüíneos deve ser um fator importante também na formação e desenvolvimento do processo ateromatoso nos enxertos. $E$, que a maior presença de dermatam sulfato na parede da veia safena resulta na sua maior susceptibilidade a ateriosclerose em relação aos enxertos arteriais.

RBCCV 44205-474

Marquezini M V, Dallan L A O, Toledo O M S - Comparative distribution of glycosaminoglycans in arteries and veins of different mammals. Rev Bras Cir Cardiovasc 1999; 14 (4): 325-30.

ABSTRACT: A comparative analysis of the glycosaminoglycan distribution in arteries and veins of humans, rats and dogs was realized. The results showed that the glycosaminoglycan distribution of the arteries was similar to that of venous tissues, where dermatan sulfate was the main glycosaminoglycan found. However, the proportion of dermatan sulfate is significantly greater in venous than in arterial tissues, in the three species. The total amount of the glycosaminoglycans was significantly higher in arteries than in veins, and the highest contents were found in the aortas. These increases may be associated with structural differences of the wall of these two types of blood vessels walls. The blood pressure is significantly lower in venous tissues and veins may exhibit less compressibility than arterial. These findings open perspectives for a better understanding of biochemical changes that could be related to the progressive degenerative vascular process, especially in the structural changes that saphenous veins undergo, when used as grafts in myocardial revascularization.

DESCRIPTORS: Glicosaminoglycans, analysis. Arteries, chemistry. Veins, chemistry.

AGRADECIMENTOS: Esta pesquisa foi apoiada por: CNPq, FAPESP e Fundação Pró-Sangue de São Paulo.

\section{REFERÊNCIAS BIBLIOGRÁFICAS}

1 Wight T N - Proteoglycans in pathological conditions: atherosclerosis. Fed Proc 1985; 49: 381-5.

2 Berenson G S, Radhakrisnamurthy B, Dalferes E R Jr, Srinivasan S R - Carbohydrate macromolecules and atherosclerosis. Human Pathol 1974; 2: 57-62.

3 Wagner W D, Salisbury B G - Aortic total glycosaminoglycan and dermatan sulfate changes in atherosclerotic Rhesus monkeys. Lab Invest 1978; 39: 328-32.

4 Toledo O M S \& Mourão P A S - Sulfated glycosa- minoglycans in normal aortic wall of different mammals. Artery 1980; 6: 341-53.

5 Camejo G - The interaction of lipids and lipoproteins with intercellular matrix of arterial tissue: its possible role in atherogenesis. Adv Lipid Res 1982; 19: 1-53.

6 Ylä-Herttuala S, Sumuvuori H, Karkola K, Möttönen M, Nikkari T - Glycosaminoglycans in normal and atherosclerotic human coronary arteries. Lab Invest 1986; 54: 402-7.

7 Dalferes E R Jr, Radhakrishnamurthy B, Ruiz H A, Berenson G S - Composition of proteoglycans from human atherosclerotic lesions. Exp Mol Pathol 1987; 47: 363-76. 
Marquezini M V, Dallan L A O, Toledo O M S - Distribuição comparativa dos glicosaminoglicanos em artérias e veias de diferentes mamíferos. Rev Bras Cir Cardiovasc 1999; 14 (4): 325-30.

8 Hollmann J, Schmidt A, Bassewitz D B, Buddecke E Relationship of sulfated glycosaminoglycans and cholesterol content in normal and atherosclerotic human aorta. Arteriosclerosis 1989; 9: 154-8.

9 Hunter G C, Dubick M A, Keen C L, Eskelson C D Effects of hypertension on aortic antioxidant status in human abdominal aneurysmal and occlusive diseases. Proc Soc Exp Biol Med 1991; 196: 273-9.

10 Robertson W B \& Strong J P - Atherosclerosis in persons with hypertension and diabetes mellitus. Lab Invest 1968; 18: 538-51.

11 Ichida T \& Kalant N - Aortic glycosaminoglycans in atheroma and alloxan diabetes. Can J Biochem 1968; 46: $249-60$

12 Sirek O V, Sirek A, Cukerman E - Arterial glycosaminoglycans in diabetic dogs. Blood Vessels 1980; 17: $271-5$

13 Wasty F, Alavi M Z, Moore S - Distribution of glycosaminoglycans in the intima of human aortas: changes in atherosclerosis and diabetes mellitus. Diabetologia 1993; 36: 316-22.

14 Manley G \& Hawksworth $\mathrm{J}$ - Distribution of mucopolysaccharides in the human vascular tree. Nature 1965; 206: 1152-3.

15 Murata $\mathrm{K}$ \& Yokoyama $Y$ - Acidic glycosaminoglycans in human atherosclerotic cerebral arterial tissues. Atherosclerosis 1989; 78: 69-79.

16 Cardoso L E M, Erlich R B, Rudge M C, Peraçoli J C, Mourão P A S - A comparative analysis of glycosaminoglycans from human umbilical arteries in normal subjects and in pathological conditions affecting pregnancy. Lab Invest 1992; 67: 588-95.

17 Izuka K \& Murata K - Inhibitory effects of human aortic and venous acid glycosaminoglycans on thrombus formation. Atherosclerosis 1972; 16: 217-24.

18 Marquezini M V, Strunz C M, Dallan L A, Toledo O M - Glycosaminoglycan distribution in atherosclerotic saphenous vein grafts. Cardiology 1995; 86: 143-6.

19 Walton K W, Slaney G, Ashton F - Atherosclerosis in vascular grafts for peripheral vascular disease: I. Autogenous vein grafts. Atherosclerosis 1985; 54: 49-64.

20 Grondin C M, Campeau L, Lespérance J - Atherosclerotic changes in coronary vein grafts six years after operation: angiographic aspect in 110 patients. $J$ Thorac Cardiovasc Surg 1979; 77: 24-31.

21 Soyombo A A, Angelini G D, Bryan A J, Newby A C - Surgical preparation induces injury and promotes smooth muscle cell proliferation in a culture of human saphenous vein. Cardiovasc Res 1993; 27: 1961-7.

22 Riessen R, Isner J M, Blessing E, Loushin C, Nikol S, Wight T N-Regional differences in the distribution of proteoglycans biglycan and decorin in the extracelular matrix of atherosclerotic and restenotic human coronary arteries. Am J Pathol 1994; 144: 962-74.

23 Hoch J R, Stark V K, Turnipseed W D - The temporal relationship between the development of vein graft intimal hyperplasia and growth factor gene expression. J Vasc Surg 1995, 22: 51-8.

24 Lin $\mathrm{H}$, Wilson J E, Roberts C R et al. - Biglycan, decorin, and versican protein expression patterns in coronary arteriopathy of human cardiac allografts: distinctness as compared to native atherosclerosis. J Heart Lung Transplantat 1996; 15: 1233-47.

25 Dilley R J, McGeachie J K, Prendergast F J - A review of the histologic changes in vein-to-artery grafts, with particular reference to intimal hyperplasia. Arch Surg 1988; 123: 691-6.

26 Davies M G \& Hagen P O - Pathobiology of intimal hyperplasia. Br J Surg 1994; 81: 1254-69.

27 Scott L, Kerr A, Haydock D, Merrilees M - Subendotelial proteoglycan synthesis and transforming growth factor beta distribution correlate with susceptbility to atherosclerosis. J Vas Res 1977; 34: 365-77.

28 Ruoslahti E - Proteoglycans in cell regulation. J Biol Chem 1989; 264: 13369-72.

29 Dietrich C P \& Dietrich S M - Electrophoretic behaviour of acidic mucopolysaccharides in diamine buffers. Anal Biochem 1976; 70: 645-7.

30 Saito H, Yamagata Y, Suzuki S - Enzymatic methods for the determination of small quantities of isomeric chondroitin sulfates. J Biol Chem 1968; 243: 1536-42.

31 Wegrowski J, Moczar M, Robert L - Proteoglycans from pig aorta: comparative study of their interactions with lipoproteins. Biochem J 1986; 235: 823-31.

32 Sambandam T, Baker J R, Christner J E, Ekborg S L Specificity of the low density lipoprotein-glycosaminoglycan interaction. Arterioscler Thromb 1991; 11: 561-8.

33 O' Brien K D, Olin K L, Alpers C E et al. - Comparison of apolipoprotein and proteoglycan deposits in human coronary atherosclerotic plaques: colocalization of biglycan with apolipoproteins. Circulation 1998; 98: 519-27.

34 Pentikäinen M O, Öörni K, Lassila R, Kovanen P T - The proteoglycan decorin links low density lipoproteins with collagen type I. J Biol Chem 1997; 272: 7633-8. 